\title{
Personalised therapeutic and dietary interventions as an alternative non-invasive management of temporal lobe epilepsy
}

Elika Garg ${ }^{1 *}$, Sandhya Koche ${ }^{1,2}$, Saher Mehdi ${ }^{1^{*}}$

${ }^{1}$ wellOwise Research and Development, IIITD Innovation \& Incubation Centre, Okhla Industrial Estate Phase 3 Rd, Shyam Nagar, Okhla Industrial Area, New Delhi, Delhi 110020

2Department of Neurology, Sri Balaji Action Medical Institute, A 4 Block, Paschim Vihar, New Delhi, Delhi - 110063

*Corresponding authors : saher@wellowise.com; elika@wellowise.com

\begin{abstract}
A 12 year old boy was diagnosed with temporal lobe epilepsy (TLE) along with mesial temporal sclerosis based on MRI (magnetic resonance imaging) results. The diagnosis was further confirmed by genetic analysis. He also had minor psychiatric symptoms of obsessive-compulsive disorders and mood swings. After 4 years of treatment with Sodium Valproate no change in symptoms was observed. Genetic testing along with deep phenotyping revealed altered glutamate pathway and metabolism. Post genetic testing the patient was put on a combination of Sodium Valproate and Valproic acid along with supplementation of $\mathrm{N}$-acetylcysteine (NAC), Cyanocobalamin, Pyridoxine and Cholecalciferol. Within three months of this combined therapy the patient experienced complete elimination of seizures and drastic improvement in mood and social behaviour. The case report highlights the importance of precision diagnosis in understanding the underlying perturbed pathways in structural epilepsy like TLE and demonstrates the importance of non-invasive targeted therapy in such cases.
\end{abstract}

Keywords: Precision diagnosis, Personalised interventions, Temporal lobe epilepsy (TLE) 


\section{Introduction}

Temporal lobe epilepsy (TLE) is the most common form of focal (partial) epilepsy. Anti-epileptic $(A E)$ medications often fail to make people seizure free but may help to reduce the frequency of seizures. Surgical resection of mesial temporal regions is often needed to ensure seizure-free life [1]. Although the molecular mechanisms underlying TLE are quite well studied now [2, 3, 4, 5], genetic testing for the same is far from the norm in clinical practice. Here we demonstrate that understanding genetic risk factors of a patient that may contribute to alteration like neuronal death and birth, gliosis, dendritic sprouting, molecular reorganisation of membrane and extracellular matrix proteins and other intermediates involved in cellular homeostasis can provide vital insights in TLE management. It can have a positive cascading effect as precise diagnosis of seizure-subtype leads to better prognosis and guides therapeutic modifications of seizure-induced molecular and cellular responses. Altogether, it offers an opportunity for better interventions beyond surgery and AE drugs.

\section{Case presentation}

Patient is a 12 year old boy who has presented with epileptic seizures since four years prior. His MRI (magnetic resonance imaging) report indicated atrophy of the right hippocampus with hyperintense signal intensity within it on T2W \& FLAIR (T2-weighted \& Fluid-Attenuated Inversion Recovery) images and prominent temporal horn of right lateral ventricle as compared to left side scan of right mesial temporal sclerosis. Patient was diagnosed with mesial temporal lobe epilepsy and was taking Sodium Valproate (200 mg) for four years. Consulting clinician referred him for precision diagnosis testing which involves deep phenotyping and comprehensive genetic screening for epilepsy and related comorbidities.

\section{Deep Phenotyping}

Detailed case history of the patient and blood marker profiling data was collected as part of deep phenotyping. Patient was found to have no known family history of epilepsy 
or any other neurological condition. His parents observed a lack of social bonding with his peer group, concentration-deficit, mood swings and anger issues. They also noticed an abnormal hand and leg automatism as well as ictal whistling during sleep. Blood markers profiling of the patient revealed that he had extremely low levels of vitamin $D$ and sub-optimal levels of vitamin B12 [Table 1].

\section{Genotyping}

Comprehensive clinical genetic testing was primarily sought to identify single nucleotide polymorphisms (SNPs) in key epilepsy genes that could be contributing to seizures. The Illumina Infinium Global Screening Array-24 v2.0 BeadChip was used for this purpose. No ClinVar-designated [6] pathogenic SNPs were found in epileptic pathways related to TLE or seizure disorder. Supplementary Table 1 lists all the genes that were investigated.

However, several variations were found in key genes [Table 2] associated with seizure disorders, as well as, glutamate transport and metabolism, which can confer a higher risk of TLE. Furthermore, heterozygous ClinVar-designated pathogenic SNPs were found in ALDH7A1 (aldehyde dehydrogenase 7 family member A1) and CBS (cystathionine beta-synthase) genes (rs372660425 and rs5742905, respectively), which were also SIFT-designated [7] deleterious, and had low allele-frequencies. Both mutations are associated with pyridoxine (vitamin B6) levels : rs372660425 mutation can lead to pyridoxine-dependent epilepsy, and rs5742905 mutation can lead to pyridoxine-responsive homocystinuria due to CBS deficiency. Supplementary Table 2 lists important mutations that were investigated.

\section{Polygenic Risk Scoring (PRS)}

We further assessed general polygenic risks in the patient that may help to guide the treatment regime. Patient was flagged for risk of vitamin B12 deficiency, but no risk was reported for either vitamin D or vitamin B6. Notably, carbohydrate and protein metabolism were found to be at risk of being dysfunctional.

Here, PRS risk implies unusually high levels of mutations in associated genes. 


\section{Management and Outcome}

Patient was presented with typical symptoms of TLE with marked hippocampus shrinkage and mesial temporal sclerosis confirmed by MRI. Deep phenotyping and genetic testing highlighted the underlying vitamin deficiencies, flawed food responses, and altered glutamate transport and metabolism. Hence, in addition to a combination of AE medication (Sodium Valproate and Valproic acid), therapeutic and dietary recommendations were made to obviate the underlying altered molecular responses.

\section{Therapeutic and Dietary Recommendations}

The patient was advised vitamin D supplements to combat the low levels observed in the blood markers report, vitamin B12 supplements to help overcome the genetic risk of deficiency, and vitamin B6 supplements to prevent pyridoxine-dependent pathogenicity. $\mathrm{N}$-acetylcysteine (NAC) was also recommended to aid epilepsy treatment. Furthermore, a ketogenic diet plan rich in healthy fats, vitamins and minerals was suggested to assist carbohydrate and protein metabolism. The patient was asked to comply with these recommendations for six months, followed by a repeat checkup by the referring clinician.

\section{Outcome}

Complete elimination of seizures was observed following the personalised therapeutic and dietary recommendation in addition to the $A E$ drug therapy. No ictal symptoms were present after 3 months and marked improvement in social and behavioral symptoms was observed after 6 months. 


\section{Discussion}

The refractory and drug-resistant nature of temporal lobe epilepsy, with pathologically and radiologically proven mesial lobe sclerosis and hippocampus shrinkage makes surgical resection the only viable option in most cases of TLE [8]. But in some cases seizure may return even after surgery. A longitudinal study cohort study $(n=85)$ on mild mesial temporal lobe epilepsy demonstrated that one quarter of patients became resistant to medication post surgery and presence of hippocampal sclerosis further increased the likelihood of refractoriness [9]. Several research publications have reiterated the importance of understanding the underlying pathophysiology [1] and molecular mechanisms [10], even in typical structural epilepsy like TLE. Prevention and reversal of hippocampal damage along with understanding of molecular events leading to epileptogenesis can help find newer and more effective therapeutic options for treatment of TLE.

The patient described here is a poster child for TLE, with typical physical symptoms like ictal whistling, hand and leg automatisms that are confirmed by MRI images showing early signs of hippocampal shrinkage and mesial sclerosis. After four years of $A E$ therapy with Sodium Valproate, no change in his symptoms was observed. In absence of surgery as an option owing to his young age, precision diagnosis helped the clinician and genetic counsellor take a more informed approach to try newer therapy guided by molecular changes observed in the patient.

Deep phenotyping indicated severe vitamin $D$ deficiency in the child. We suspect this was caused by the interference in vitamin $D$ absorption that some antiseizure medications can produce [11]. Correction of vitamin $D$ deficiency has been shown to lower the frequency of seizure, especially in younger epilepsy patients [12, 13]. Incidence of vitamin deficiency is reported to be as high as $90 \%$ in Indian children due to predominantly vegetarian diet and lack of seasonal exposure to sunlight [14]. 
Exhaustive genetic analysis revealed that the patient has several variations in two members of GABA(B) receptor subunit, GABBR1 (Gamma-Aminobutyric Acid Type B Receptor Subunit 1) and GABBR2 (Gamma-Aminobutyric Acid Type B Receptor Subunit 2). Gene polymorphism in these receptors have been implicated in temporal lobe epilepsy and mesial temporal lobe epilepsy [15, 16]. We also found variations in GAD1 (Glutamate Decarboxylase 1), GLUL (Glutamate-Ammonia Ligase), GRIK1(Glutamate Ionotropic Receptor Kainate Type Subunit 1) and GRIN2B (Glutamate lonotropic Receptor NMDA Type Subunit 2B). Enzyme encoded by GAD1 is responsible for catalyzing the production of GABA (Gamma-Aminobutyric Acid) from $L$ glutamic acid. Defects in GAD1 activity and function have been associated with temporal lobe epilepsy [17], while GRIK1 and GRIN2B encode for ionotropic receptors that contribute to excitatory postsynaptic currents. Several naturally occurring variations in GRIN2B have been associated with autism spectrum disorder, schizophrenia, and epilepsy.

Additionally, several variations associated with glutamate transporter family members like SLC1A1 (Solute Carrier Family 1 Member 1), SLC1A2 (Solute Carrier Family 1 Member 2) and SLC1A3 (Solute Carrier Family 1 Member 3) were identified. These high-affinity glutamate transporters play an essential role in transporting glutamate across plasma membranes.

Lastly, mutations associated with LGI1 (Leucine rich glioma inactivated 1) and RELN (Reelin) are also important for this case, because both genes are implicated in TLE and its associated psychiatric symptoms.

Genetic analysis clearly indicated a defective glutamate metabolism and transport underlying the symptoms of TLE in the patient. In order to fix the defective glutamate metabolism, NAC supplementation was recommended to the patient. Several clinical studies have indicated that NAC can be useful in treating neurological disorders by modulating glutamate dysregulation [18, 19]. Also, cobalamin and pyridoxine supplementation [20] was given to overcome the vitamin deficiencies as indicated by 
both blood test and polygenic risk analysis. Ketogenic diet modifications were also suggested due to the altered protein and carbohydrate metabolism as indicated by elevated genetic risk associated with these two traits. Increasing amounts of evidence support the positive role of a ketogenic diet in epilepsy management [21].

Following six months of personalised dietary and therapeutic recommendation complete absence of seizures and drastic improvement in related psychiatric symptoms was observed in the patient The case report clearly demonstrates the importance of precision diagnosis and personalised intervention in effective management of refractory and drug-resistant epilepsy. It also serves as an attractive non-invasive alternative to surgery. 
Tables

Table 1. Blood markers profile of patient showing severe vitamin D deficiency.

\begin{tabular}{|l|l|l|l|}
\hline Test Name & Result & Unit & Reference Range \\
\hline Homocysteine; Quantitative, Serum & 9.82 & umol/L & $5.46-16.20$ \\
\hline $\begin{array}{l}\text { Vitamin B12; Cyanocobalamin, } \\
\text { Serum }\end{array}$ & 767 & $\mathrm{pg} / \mathrm{mL}$ & $211.00-911.00$ \\
\hline Vitamin D, 25-Hydroxy, Serum & 11.89 & $\mathrm{nmol} / \mathrm{L}$ & $75.00-250.00$ \\
\hline Hemoglobin; Hb, Blood & 13.90 & $\mathrm{gm} / \mathrm{dL}$ & $11.50-15.50$ \\
\hline
\end{tabular}

Table 2. Key genes affecting molecular responses in the patient

\begin{tabular}{|l|l|}
\hline Key genes & Related traits \\
\hline $\begin{array}{l}\text { GABBR1, GABBR2, GAD1, GLUL, GRIK1, } \\
\text { GRIN2B, LGI1, SLC1A1, SLC1A2, SLC1A3, } \\
\text { RELN }\end{array}$ & $\begin{array}{l}\text { Glutamate transport and } \\
\text { metabolism; temporal lobe epilepsy }\end{array}$ \\
\hline DIAPH1, HMGCR, KCNQ3, SCN1A, QPRT & Seizure disorder \\
\hline MECP2, MTR, NTRK2, BDNF & $\begin{array}{l}\text { Mental health; metabolic diseases; } \\
\text { inflammatory conditions }\end{array}$ \\
\hline
\end{tabular}




\section{References}

1. Curia G, Lucchi C, Vinet J, Gualtieri F, Marinelli C, Torsello A, Costantino L, Biagini, G. Pathophysiogenesis of Mesial Temporal Lobe Epilepsy: Is Prevention of Damage Antiepileptogenic? Current Medicinal Chemistry. 2014 Jul; 21: 663-688. doi: 10.2174/0929867320666131119152201. PMID: 24251566.

2. Staley K. Molecular mechanisms of epilepsy. Nature neuroscience. 2015 Feb 24; 18(3): 367-372. doi: 10.1038/nn.3947. PMID: 25710839.

3. Brooks-Kayal AR, Shumate MD, Jin H, Rikhter TY, Coulter DA. Selective changes in single cell $\mathrm{GABA}(\mathrm{A})$ receptor subunit expression and function in temporal lobe epilepsy. Nat Med. 1998 Oct;4(10):1166-72. doi: 10.1038/2661. Erratum in: Nat Med 1999 May;5(5):590. PMID: 9771750.

4. Schenkel LC, Bragatti JA, Becker JA, Torres CM, Martin KC, de Souza AC, Manfro GG, Leistner-Segal S, Bianchin MM. Serotonin gene polymorphisms and psychiatry comorbidities in temporal lobe epilepsy. Epilepsy Res. 2012 May;99(3):260-6. doi: 10.1016/j.eplepsyres.2011.12.005. Epub 2012 Jan 4. PMID: 22222329.

5. Eid T, Thomas MJ, Spencer DD, Rundén-Pran E, Lai JC, Malthankar GV, Kim JH, Danbolt NC, Ottersen OP, de Lanerolle NC. Loss of glutamine synthetase in the human epileptogenic hippocampus: possible mechanism for raised extracellular glutamate in mesial temporal lobe epilepsy. Lancet. 2004 Jan 3;363(9402):28-37. doi: 10.1016/s0140-6736(03)15166-5. PMID: 14723991.

6. Landrum MJ, Lee JM, Benson M, Brown GR, Chao C, Chitipiralla S, Gu B, Hart J, Hoffman D, Jang W, Karapetyan K, Katz K, Liu C, Maddipatla Z, Malheiro A, McDaniel K, Ovetsky M, Riley G, Zhou G, Holmes JB, Kattman BL, Maglott DR. ClinVar: improving access to variant interpretations and supporting evidence. Nucleic Acids Research. 2017 Nov 20; 46(Database issue): D1062-D1067. doi: 10.1093/nar/gkx1153. PMID: 29165669 
7. Sim NL, Kumar P, Hu J, Henikoff S, Schneider G, Ng PC. SIFT web server: predicting effects of amino acid substitutions on proteins. Nucleic Acids Research. 2012 Jun 9; 40(Web Server issue): W452-W457. doi: 10.1093/nar/gks539. PMID: 22689647

8. Jobst BC, Cascino GD. Resective epilepsy surgery for drug-resistant focal epilepsy: a review. JAMA. 2015 Jan 20;313(3):285-93. doi: 10.1001/jama.2014.17426. PMID: 25602999.

9. Jobst BC. It Goes Downhill From Here but Do Not Despair: Mesial Temporal Lobe Epilepsy Is a Progressive Disease, but It Can Be Benign. Epilepsy Currents. 2016 Nov-Dec; 16(6): 380-381. doi: 10.5698/1535-7511-16.6.380. PMID: 27857615

10. Pitkänen A, Sutula TP. Is epilepsy a progressive disorder? Prospects for new therapeutic approaches in temporal-lobe epilepsy. Lancet Neurol. 2002 Jul;1(3):173-81. doi: 10.1016/s1474-4422(02)00073-x. PMID: 12849486.

11. Menon B, Harinarayan CV. The effect of anti epileptic drug therapy on serum 25-hydroxyvitamin $\mathrm{D}$ and parameters of calcium and bone metabolism--a longitudinal study. Seizure. 2010 Apr;19(3):153-8. doi: 10.1016/j.seizure.2010.01.006. Epub 2010 Feb 7. PMID: 20144552.

12. Holló A, Clemens Z, Kamondi A, Lakatos $P$, Szücs A. Correction of vitamin D deficiency improves seizure control in epilepsy: a pilot study. Epilepsy Behav. 2012 May;24(1):131-3. doi: 10.1016/j.yebeh.2012.03.011. Epub 2012 Apr 11. PMID: 22503468.

13. Khalifah RA, Hudairi A, Homyani DA, Hamad MH, Bashiri FA. Vitamin D supplementation to prevent vitamin $D$ deficiency for children with epilepsy: Randomized pragmatic trial protocol. Medicine (Baltimore). 2018 Oct;97(40):e12734. doi: 10.1097/MD.0000000000012734. PMID: 30290685.

14. Angurana, Suresh. Vitamin D deficiency in children: Is there a need for routine supplementation? Indian Journal of Endocrinology and Metabolism (2018) 22(5):714-715. doi: 0.4103/ijem.IJEM_215_18. PMID: 30294588 
15. Wang X, Sun W, Zhu X, Li L, Wu X, Lin H, Zhu S, Liu A, Du T, Liu Y, Niu N, Wang Y, Liu Y. Association between the gamma-aminobutyric acid type $B$ receptor 1 and 2 gene polymorphisms and mesial temporal lobe epilepsy in a Han Chinese population. Epilepsy Res. 2008 Oct;81(2-3):198-203. doi: 10.1016/j.eplepsyres.2008.06.001. Epub 2008 Jul 23. PMID: 18653317.

16. Xi B, Chen J, Yang L, Wang W, Fu M, Wang C. GABBR1 gene polymorphism (G1465A) is associated with temporal lobe epilepsy. Epilepsy Res. 2011 Sep;96(1-2):58-63. doi: 10.1016/j.eplepsyres.2011.04.014. Epub 2011 May 28. PMID: 21621395.

17. Obata A, Kutoku Y, Sunada Y, Okauchi S, Kimura T, Hirukawa H, Tanabe A, Kinoshita T, Kohara K, Tatsumi F, Shimoda M, Kamei S, Nakanishi S, Mune T, Kaku K, Kaneto H. Temporal lobe epilepsy associated with GAD autoimmunity. Acta Diabetol. 2017 Mar;54(3):321-323. doi: 10.1007/s00592-016-0910-9. Epub 2016 Sep 8. PMID: 27638303.

18. Deepmala, Slattery J, Kumar N, Delhey L, Berk M, Dean O, Spielholz C, Frye R. Clinical trials of $\mathrm{N}$-acetylcysteine in psychiatry and neurology: A systematic review. Neurosci Biobehav Rev. 2015 Aug;55:294-321. doi: 10.1016/j.neubiorev.2015.04.015. Epub 2015 May 6. PMID: 25957927.

19. Dean O, Giorlando F, Berk M. N-acetylcysteine in psychiatry: current therapeutic evidence and potential mechanisms of action. J Psychiatry Neurosci. 2011 Mar;36(2):78-86. doi: 10.1503/jpn.100057. PMID: 21118657.

20. Kim JE, Cho KO. Functional Nutrients for Epilepsy. Nutrients. 2019 Jun 10;11(6):1309. doi: 10.3390/nu11061309. PMID: 31185666.

21. D'Andrea Meira I, Romão TT, Pires do Prado HJ, Krüger LT, Pires MEP, da Conceição PO. Ketogenic Diet and Epilepsy: What We Know So Far. Front Neurosci. 2019 Jan 29;13:5. doi: 10.3389/fnins.2019.00005. PMID: 30760973 